\title{
Performance Analysis of a Dynamic Bandwidth Allocation Algorithm in a Circuit-Switched Communications Network
}

\author{
Timothy M. Schwamb, Rusty O. Baldwin, Richard A. Raines, Michael A. Temple
}

\begin{abstract}
Military communications networks typically employ a gateway multiplexer to aggregate all communications traffic onto a single link. These multiplexers typically allocate bandwidth statically via time-division multiplexing (TDM). Inefficiencies occur when a high-bandwidth circuit, e.g., a video teleconferencing circuit, is inactive rendering a considerable portion of the aggregate bandwidth wasted. Dynamic bandwidth allocation (DBA) reclaims unused bandwidth from circuits with low utilization and reallocates it to circuits with higher utilization without adversely affecting queuing delay. The DBA algorithm developed here measures instantaneous utilization by counting frames arriving during the transmission time of a single frame on the aggregate link. The maximum calculated utilization observed over a monitoring period is used to calculate the bandwidth available for reallocation.

Utilization results indicate the proposed DBA algorithm significantly outperforms a static allocation model in all cases. The best configuration uses a 65536 bps allocation granularity and a 10 second monitoring period. Utilization gains observed with this configuration were almost $17 \%$ over the static allocation method. Queuing delays increased by $50 \%$ but remained acceptable, even for real-time traffic.
\end{abstract}

Keywords- Dynamic Bandwidth Allocation, CircuitSwitched Communications Network, Time-Division Multiplexing, Military Tactical Communications Network.

\section{INTRODUCTION}

$\mathrm{M}$ ILITARY communications networks employ a gateway multiplexer to aggregate all communications traffic onto a single link. These multiplexers typically allocate bandwidth statically using time-division multiplexing (TDM). When a high-bandwidth circuit, such as a video teleconferencing (VTC) circuit is rarely active, however, a considerable portion of the bandwidth is wasted. Dynamic bandwidth allocation (DBA) reclaims unused bandwidth from circuits with low utilization and reallocates it to a circuit with high utilization without adversely affecting queuing delay [8].

\section{RELATED WORK}

Dynamic bandwidth allocation has enjoyed much attention, especially in the ATM arena. ATM is popular because it classifies traffic based on arrival characteristics and endto-end delay requirements. Many DBA algorithms rely on a measure of instantaneous loading levels [6], [7], [8]. Shioda proposes to measure a circuit's demand for bandwidth based on an estimation of the circuit's blocking probability [7]. This is an effective metric because the blocking probability will increase as available bandwidth is depleted, thus indicating a greater bandwidth demand. However, the blocking probability computations for this algorithm differ by traffic class and most are extremely complex. Saito's

The views expressed in this paper are those of the author and do not reflect the official position of the US Air Force, Department of Defense or the US Government. approach is much simpler, using a simple cell count to measure the bandwidth in use [6]. His algorithm divides the bandwidth into usage levels where the number of cells that arrive during a measurement period determines the level of bandwidth usage. However, the algorithm only takes into account the previous bandwidth in use so a sudden increase in the loading level may cause degraded performance in quality of service guaranteed circuits. A similar but more robust algorithm developed for ATM was devised by Shiomoto, Chaki, and Yamanaka [8]. They propose to allocate bandwidth dynamically based on a measurement of instantaneous virtual path utilization. This algorithm was chosen for this study above others because, like Saito's algorithm, it relies on a simple cell count to determine the instantaneous utilization, but it uses the current measurement and an amalgamation of previous measurements. It also fits nicely into a TDM environment and can be fully implemented in software.

\section{A. Algorithm Overview}

The instantaneous virtual path utilization is defined as

$$
\rho(t)=\sum_{i} \frac{R_{i}(t)}{C}
$$

where $R_{i}(t)$ is the peak cell rate of the $i$ th circuit at time $t$ and $C$ is the channel capacity. In order to estimate this instantaneous utilization, the number of cells arriving during one cell transmission period are counted. This cell count is sent through an exponential averager to provide an estimate of the instantaneous virtual path utilization. The exponential average, $\hat{\rho}_{i}$, is

$$
\hat{\rho}_{i}=\alpha n_{i}+(1-\alpha) \hat{\rho}_{i-1}, \quad 0 \leq \alpha \leq 1
$$

where, $n_{i}$ is the number of cells that arrive in the $i$ th cell transmission period, $\hat{\rho}_{i-1}$ is the estimate from the $i$-1th period, and $\alpha$ is a weighting factor, discussed below.

The utilization is tracked for a monitoring period, $T_{m}$. The maximum value of $\hat{\rho}$ observed during $T_{m}, \hat{\rho}_{\text {max }}$, serves as the basis for the admission criteria. If the new circuit is accepted, the virtual path utilization estimate is updated; if rejected, $\hat{\rho}_{\max }$ remains the same.

\section{B. Weighting Factor $\alpha$}

The value of $\alpha$ determines whether the current measurement or past measurements are more heavily weighted in the utilization estimate. As $\alpha$ approaches 1, current measurements become more significant. Conversely, as $\alpha$ approaches 0 , the amalgamation of past measurements becomes more significant. Because of the bursty nature of most circuits, it would typically be better to make $\alpha$ closer 
to 0 . For example, simulation results from Shiomoto, et al., indicate that an $\alpha$ of $4.156 \mathrm{E}-3$ produces a very accurate estimate of the system's instantaneous utilization for a virtual circuit with a peak rate of $10 \mathrm{Mbps}$ [8]. In general, $\alpha$ can be determined by

$$
\alpha=\frac{-2(1-K)+\sqrt{4(1-K)^{2}+8\left(\varepsilon^{-1}-1\right)(1-K)}}{2\left(\varepsilon^{-1}-1\right)}
$$

where $\varepsilon$ is the objective cell loss rate, $K=\cos \left(2 \pi f_{0} \Delta\right), f_{0}$ is the circuit's peak cell rate, and $\Delta$ is the time necessary to transmit a single cell on the aggregate link.

\section{Monitoring Period $T_{m}$}

The monitoring period should be sufficiently long to keep the cell loss rate below its target value. According to [8], this value should be on the order of 100 seconds. If necessary, this period can be reduced but will result in sacrificing approximately $20 \%$ of the assignable channel capacity. For a. more detailed explanation of $T_{m}$ selection refer to [8].

\section{Advantages and Disadvantages}

This algorithm has several strengths. First, it is very simple. Once the utilization estimate is obtained for the monitoring period, the admission control mechanism need only compare the requested peak rate of the new circuit to the residual bandwidth. If the requested value is less than the residual value, the circuit is accepted; otherwise it is rejected. Second, because the algorithm relies on a simple cell count to calculate the instantaneous utilization, it can be completely implemented in software. Therefore, it is possible to port this algorithm to other platforms including TDM.

A couple of disadvantages do exist. First, the algorithm is easy to implement since it assumes homogeneous circuits and peak rates. This assumption does not hold in practice. Therefore, either $\alpha$ must be dynamically adjustable, or multiple exponential averagers are required - one for each circuit. While this problem is not insurmountable, it raises the implementation cost. Second, the algorithm assumes that circuits are dynamically connected and disconnected, thus creating a dynamic amount of available bandwidth. However, in a military communications network, many circuits are persistent. Therefore, it would be advantageous to adjust previously allocated bandwidth dynamically. This difficulty is overcome by allocating bandwidth between circuits.

\section{IMPLEMENTATION}

\section{A. System Design}

Our DBA algorithm is an enhancement of that developed by Shiomoto, et al. [8]. We modify Shiomoto to support heterogeneous permanent circuits in a TDM environment.

Because the TDM system accepts heterogeneous circuits, each circuit's instantaneous utilization is calculated separately. The instantaneous utilization is calculated at the end of every time slot. At this time, the maximum and minimum utilized circuits are determined. At the end of the monitoring period, the bandwidth is adjusted. The circuit with the lowest utilization transfers all of its unused time slots (up to a specified threshold) to the circuit with the highest utilization. All other circuits remain unchanged. In the event that all circuits are utilizing all of their time slots, but those slot assignments are not that of the originally-specified rates, the time slot allocations are reset to the original assignments.

The algorithm also implements two additional features. First, a priority for CBR circuits is implemented. If the video circuit is rarely active, the DBA algorithm is unable to adjust quickly enough upon activation of the video circuit, causing input buffers to fill and queuing delays to increase. Therefore, a priority feature is implemented to ensure that the video circuit is allocated the necessary number of time slots as soon as a video frame arrives. Second, a work conservation feature is employed. Work conservation ensures that every available time slot is filled as long as at least one circuit has a frame waiting. A slot will only be empty when all input buffers are empty. For a more complete comparison, the work conservation feature was added to the static allocation model and DBA results were compared against this static model and the TDM model.

\section{B. System Configuration}

The system under test has four user circuits - a voice circuit, a video circuit, and two data circuits (labeled NIPRNET and SIPRNET). These circuits are assigned bandwidths of 262144 bps, 262144 bps, 131072 bps, and $131072 \mathrm{bps}$, respectively. The aggregate bandwidth is 786432 bps. The model assumes a fixed frame size of 4096 bits, a minimum circuit data rate of 8192 bps (32768 bps for the voice circuit), and an objective frame loss rate of $1 \mathrm{E}-4$. The DBA system is evaluated using 9 configurations - 3 monitoring periods, 3 granularities of bandwidth allocation. These 9 configurations are subjected to four loading levels. Table I lists the factor levels. The system underload is defined as one in which all circuits submit a low offered load. The three types of overload workloads are defined such that one or more circuits submit a high offered load and the remaining circuits submit a low offered load. For example, using the values from Table II, the data overload consists of the higher $93.7 \%$ offered load submitted by the data circuits and the lower $21.3 \%$ and $7.7 \%$ offered loads submitted by the voice and video circuits, respectively. Additionally, monitoring periods are much lower than the 100 seconds in [8] because initial testing showed that lower monitoring periods produce much lower queuing delays without impacting utilization gains. Performance is judged by comparing the circuits' queuing delays and the aggregate utilization of the DBA system to that of a static allocation system normally used in military communications networks. 
TABLE I

FACTORS AND Associated LeVELS

\begin{tabular}{|c|c|}
\hline Factor & Level \\
\hline \multirow{3}{*}{ Allocation Granularity } & $8192 \mathrm{bps}$ \\
& $32768 \mathrm{bps}$ \\
& $65536 \mathrm{bps}$ \\
\hline \multirow{3}{*}{ Monitoring Period } & $5 \mathrm{sec}$ \\
& $10 \mathrm{sec}$ \\
& $50 \mathrm{sec}$ \\
\hline \multirow{2}{*}{ Workload } & System Underload \\
& Data Overload \\
& Voice Overload \\
& Voice \& Data Overload \\
\hline
\end{tabular}

TABLE II

NoRMalized OfFEREd LOAD DEFINITIONS

\begin{tabular}{|c|c|c|}
\hline Circuit & $\begin{array}{c}\text { Underload } \\
\text { (Normalized) }\end{array}$ & $\begin{array}{c}\text { Overload } \\
\text { (Normalized) }\end{array}$ \\
\hline Data Circuits & $46.5 \%$ & $93.7 \%$ \\
\hline Voice Circuit & $21.3 \%$ & $42.6 \%$ \\
\hline Video Circuit & $7.7 \%$ & N/A \\
\hline
\end{tabular}

\section{Results And Analysis}

\section{A. Utilization}

Figure 1 compares the mean utilization of the DBA system to the static system. The DBA utilizations for a particular offered load represent the mean across all factors. Every DBA configuration produced statistically significant utilization gains over the static system at the $90 \%$ confidence level. The smallest average gain observed was $6.2 \%$ with the system underload. This gain shows that even in light loading, the system is still able to optimize the available bandwidth. The largest average gain observed was $20.0 \%$ with the data overload. The system performs better with the data overload than the voice and data overload because the bandwidth manager is able to optimize bandwidth allocation for the data circuits with only two circuits heavily loaded. With the voice and data overload, however, three of the four circuits are heavily loaded causing the bandwidth manager to juggle between the three circuits competing for additional bandwidth. The configuration producing the highest average utilization gains over the static system uses a 32768 bps granularity and 5 second monitoring period.

Workload accounts for $99.3 \%$ of the ovserved variation, while monitoring period and allocation granularity gave a negligible effect. This result indicates first that offered load is responsible for virtually all the utilization performance of the system. It also indicates, however, that, for a given workload, the DBA algorithm can increase utilization approximately the same irrespective of allocation granularity or monitoring period.

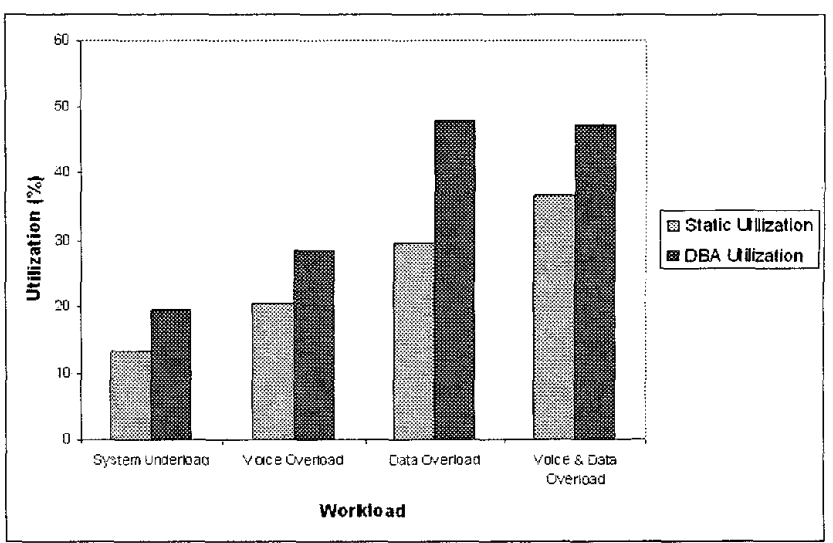

Fig. 1. Utilization: Static Allocation vs. Dynamic Allocation

\section{B. Queuing Delay}

\section{B.1 Best Configuration}

The 65536 bps allocation granularity and the 10 second monitoring period results in the lowest queuing delays for the DBA system. The 65536 bps granularity performs better because reallocations are less frequent. This, in turn, means the system does not have to adjust as often resulting in fewer times that the input buffers fill up due to a reallocation. The 10 second period performs better than the 50 second monitoring period because the 50 second period cannot react fast enough to the dynamics of the system. The 50 second period reacts much slower to a sudden increase in workload for a particular circuit, which causes increased queuing delays. The 5 second period, on the other hand, results in too many reallocations. Therefore the system cannot stabilize as quickly as with the 10 second period.

Results show that queuing delays are dramatically lower - by two orders of magnitude in most cases - over the strict TDM model. For example, video circuit queuing delay using the highest loading level with the DBA algorithm was $5.8 \mathrm{~ms}$ compared with $335 \mathrm{~ms}$ using the strict TDM algorithm. Figure 2 shows, however, that the DBA queuing delays are not always best when compared to a static system employing work conservation. In fact, the voice and data circuits' queuing delays were statistically higher on the DBA system (at 90\% confidence) than on the static system under all configurations and workloads. The video circuit's queuing delays were only statistically higher on the voice overload and voice and data overload workloads. This is due to the inclusion of the CBR priority feature. As Figure 2 shows, the voice circuit's queuing delays are significantly higher than that of the other circuits. The reason for the higher queuing delays is due to the algorithm's implementation. The algorithm looks to the next circuit in sequence to fill a potentially-unused time slot - establishing a priority among circuits. For example, the NIPRNET circuit has "first priority" on all unused time slots of the rarely-active video circuit. Because the voice circuit falls immediately before the video circuit sequentially, it has "last priority." 


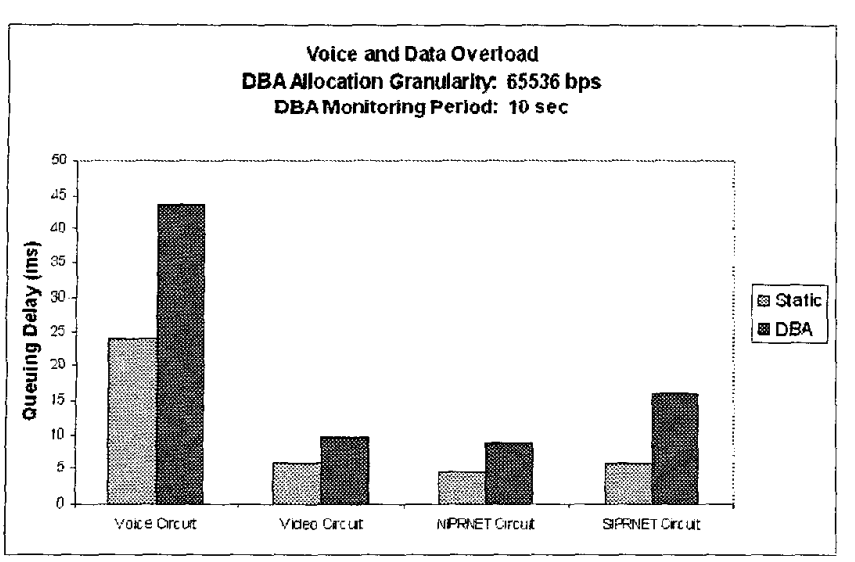

Fig. 2. Queuing Delay: Static Allocation with Work Conservation vs. DBA

The voice circuit has first priority on the SIPRNET circuit but the amount of unused time slots are much less than from the video circuit. There are two ways to eliminate this problem. The first is to ensure all circuits are prioritized based on bandwidth and delay requirements. For example, if the video circuit has the highest priority and the voice circuit has the second highest priority, then the voice circuit is given priority on the video circuit's unused time slots. The second alternative is to randomly select which circuit has "first priority" upon encountering a potentially-unused time slot. Whether this implicit priority is changed or not, the algorithm's ability to fill potentially-unused time slots clearly has a tremendous impact on the queuing delay experienced by arriving frames. Though most of the queuing delays are statistically higher than with the static allocation method, the best DBA configuration still results in acceptable end-to-end delays even with the current circuit sequencing [2].

\section{B.2 Allocation of Variation}

Workload is the single biggest contributor to the queuing delay variation observed in all four circuits, ranging from $86.1 \%$ on the voice circuit up to $97.0 \%$ on the video circuit. This result is not surprising, though. With the work conservation feature employed, the number of potentiallyempty time slots that could be filled by other circuits goes down as the offered load to each circuit increases resulting in higher queuing delays.

Each circuit is affected only minimally by allocation granularity. The voice circuit is affected the most with an observed variation of $6.7 \%$. The reason for the higher variation on the voice circuit is likely because the voice circuit must be allocated in chunks of at least $32768 \mathrm{bps}$ - the allocated bandwidth for one phone call. Consequently, an 8192 bps allocation granularity does not benefit the voice circuit like it does the other circuits, resulting in higher queuing delays. Allocation granularity accounts for $2.8 \%$ and $2.9 \%$ of the observed variation on the two respective data circuits and a statistically negligible amount on the video circuit. These results indicate that regardless of the
DBA configuration, queuing delay on these circuits is affected little by anything other than the offered load.

The monitoring period has a negligible effect on the observed variation of each circuit. In all but the video circuit's case, the monitoring period explains only slightly more of the variation than the unexplained. The monitoring period's effect on the video circuit is less than that of the unexplained variation. These results indicate that since queuing delays have been judged acceptable under the DBA algorithm, any monitoring period between 5 and 50 seconds results in acceptable queuing delays for the system.

\section{Best Overall Configuration}

Classic queuing theory states that utilization and delay are opposing metrics [3]. In other words, at some point, one metric must be sacrificed to produce significantly better results in the other. Unfortunately, utilization is usually sacrificed to keep delay low so a network can support real-time traffic such as voice or video. This is the case with dynamic bandwidth allocation. Therefore, the best overall configuration of the DBA algorithm consists of the 65536 bps allocation granularity and 10 second monitoring period. This configuration is chosen over the 32768 bps granularity and 5 second monitoring period because average utilization across all workloads is only $1.5 \%$ lower and queuing delay is the lowest of all configurations.

Under the chosen configuration and all workloads, queuing delay is low enough to meet the delay requirements for real-time traffic and utilization is increased significantly over that of the static model. The static and dynamic systems were then subjected to approximately $70 \%, 85 \%$, and $99 \%$ offered loads to determine how they perform under extreme conditions. In all cases, both systems' aggregate utilizations are only negligibly different from the offered load.

Queuing delays increase dramatically starting at the $70 \%$ loading level for both systems. Figures 3 and 4 show the resulting average queuing delays for the voice and video circuits, respectively. The queuing delay increases much faster in the dynamic system because the algorithm is still adjusting the bandwidth where possible, but minor changes in circuit activity have much more drastic effects at higher loading levels. The queuing delay increase tapers off after the $85 \%$ level (and in some cases decreases) because the system is much closer to being on continuously. In other words, most of each circuit's time slots are filled due to the high offered load reducing the slots available for reallocation to other circuits.

The system can still meet requirements, assuming that no significant congestion is encountered on the path from source to destination. As Figure 3 shows, the worst average queuing delay for the video circuit is $39.15 \mathrm{~ms}$. Data circuit queuing delays at these extreme loading levels are higher, but this is not a concern since data traffic is not held to the stringent end-to-end delay requirements of real-time traffic. If other nodes are experiencing similar congestion, however, real-time traffic might experience unacceptable delay. The circuit sequencing problem creates delays as 


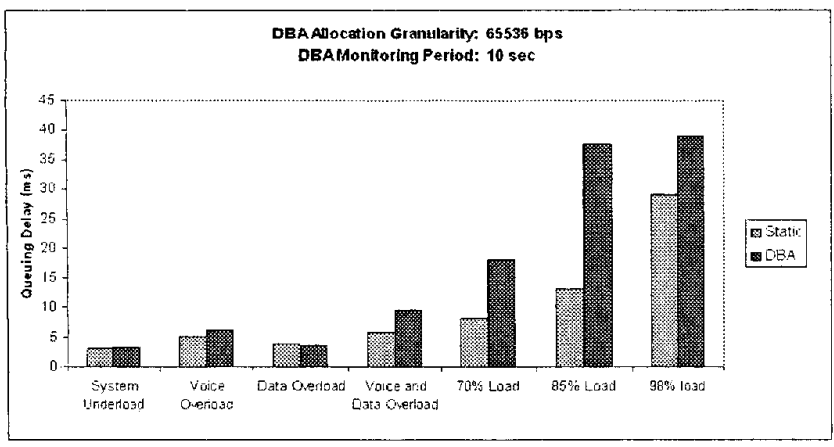

Fig. 3. Comparison of Video Circuit Queuing Delays

large as $384 \mathrm{~ms}$ on the voice circuit under extreme loading. However, implementing one of the two solutions described in Section B.1 should mitigate this excessive queuing delay. Furthermore, extreme loading conditions such as these should rarely occur. The voice and data overload represent heavy loading of voice and data circuits but extremely low loading of the video circuit. This assumes that a video teleconferencing circuit is rarely used more than an hour per day in a tactical military environment. This usage level must reverse itself for near capacity loading levels to occur. Second, the offered load on the voice circuit ceases to represent normal voice communication as observed in [1] above the $70 \%$ loading level. Therefore it is reasonable to assume that extreme loading conditions such as these could only occur for a short durations which should only minimally disrupt communications traffic by increasing delay. Under this configuration, then, the DBA algorithm keeps queuing delay sufficiently low while significantly increasing aggregate utilization.

\section{CONClusions ANd Future Work}

\section{A. Conclusions}

The inclusion of the CBR priority and work conservation features enables the dynamic bandwidth allocation algorithm to produce higher aggregate utilizations than the static allocation model in all cases. Using DBA, tactical military communications networks can bring information to the warfighter more efficiently in spite of the small satellite bandwidths allocated to deployed sites. Furthermore, the algorithm can be completely implemented in software, significantly reducing the cost of adopting DBA. The algorithm is quite general so it can be applied to multiple TDM platforms, including NET's Promina [4] - the military's primary gateway multiplexer. Additionally, the algorithm is robust enough to function independent of line speed, making it a viable option for even high-speed multiplexers. Our DBA algorithm is a powerful tool to optimize the available resources of a communications network.

\section{B. Recommendations for Future Work}

Although our DBA algorithm is robust and powerful, it is not without limitations or questions to be explained. First and most important, the delay resulting from the algorithm's calculations has not been characterized. The simulation tool, OPNET Modeler [5], uses state-transition diagrams to describe a process or model. State transitions are assumed to be instantaneous. We believe that utilization calculation and subsequent reallocations will not cause significant delay, but this assumption should be verified.

Second, the circuit sequencing issue described in Section B.1 should be resolved. Two possible solutions were presented for a single video and voice circuit - prioritizing the circuits so that real-time traffic has a higher priority on unused time slots and randomly selecting a circuit to fill an unused time slot. The system produced acceptable delays under these conditions, but other circuit configurations need to be investigated as well. For instance, if there were multiple voice circuits, the proposed circuit prioritization might not provide optimal results. Conversely, randomly choosing a circuit to fill an unused time slot might result in excessive delay due to processing overhead.

\section{REFERENCES}

[1] F. Callegati, M. Pedrelli, and C. Raffaelli. Analysis of CSMA/CD protocol for wireless networking of ATM multiservice applications. In Proceedings of 5 th International Conference on Universal Personal Communications, volume 1, pages 286-290. Institute of Electrical and Electronics Engineers, 1996.

[2] International Telecommunications Union. ITU Rec. G.114: OneWay Transmission Time, February 1996.

[3] Raj Jain. The Art of Computer Systems Performance Analysis. John Wiley and Sons, New York, NY, 1991.

[4] Network Equipment Technologies, Inc., Redwood City, CA. IDNX Theory of Operation and Diagnostics, March 1996.

[5] OPNET Technologies, Inc., Washington, DC. OPNET Modeler 8.O.C, July 2001 .

[6] Hiroshi Saito. Trials of dynamic bandwidth allocation in ATM networks. In IEEE ATM'97 Workshop Proceedings, pages 141$146,1997$.

[7] Shigeo Shioda. Self-sizing network - algorithmic aspects. International Journal of Communication Systems, 11:43-58, 1998.

[8] Kohei Shiomoto, Shinichiro Chaki, and Naoaki Yamanaka. A simple bandwidth management strategy based on measurements of instantaneous virtual path utilization in ATM networks. IEEE/ACM Transactions on Networking, 6:625-633, October 1998. 